\title{
Revisión
}

\section{Estado actual de la vacuna recombinante contra el virus del papiloma humano}

\author{
(State of the art on human papillomavirus vaccine)
}

Wendy Chan Acón', Lara Aguilar Morales', Carolina Soley',Adriano Arguedas Mohs',2

\section{Resumen}

La infección por el virus del papiloma humano (VPH) es una enfermedad de transmisión sexual común. Alrededor del mundo millones de personas están infectadas y el resto de la población en general tiene un riesgo de contraer la infección superior al $50 \%$. El virus se asocia aproximadamente a un $100 \%$ de los casos de cáncer cervical; a un 100\% de las neoplasias cervicales intraepiteliales grados 1, 2, 3; a un 40\% de los casos de cáncer de vulva, vagina y pene, $100 \%$ de las verrugas genitales; a un $100 \%$ de las papilomatosis respiratorias recurrentes; a un $90 \%$ del cáncer anal y a un $12 \%$ del cáncer de cabeza y cuello, predominantemente en orofaringe y amígdala. Actualmente, el uso de dos vacunas está aprobado en diversos países: Gardasil ${ }^{\circledR}$ y Cervarix ${ }^{\circledR}$. Ambas están compuestas por proteínas L1 de VPH, en forma de partículas no infecciosas similares al virus (VLPs) producidas por tecnología de ADN recombinante, adsorbidas en adyuvantes que contienen aluminio. La eficacia hallada en diversos estudios en sujetos no expuestos previamente al virus se encuentran en el rango del $98.8 \%$ al $100.0 \%$ para la prevención de neoplasias cervicales, vulvares y vaginales intraepiteliales, grados 2 y 3 , relacionados con el VPH-16/18, además de los adenocarcinomas in situ y verrugas genitales causadas por VPH$16 / 18 / 6 / 11$ en el caso de Gardasil ${ }^{\circledR}$ y una eficacia del $100 \%$ en el caso de Cervarix ${ }^{\circledR}$ para la prevención de neoplasias cervicales grado 2 y 3 relacionados con el VPH-16/18. La eficacia de ambas se mantiene alrededor de los 5 años. Hasta el momento no se le ha atribuido a la vacuna ningún efecto terapéutico, solo se administra con fines profilácticos, sin embargo, esta no debe ser considerada como un sustituto de las pruebas de tamizaje para la prevención del cáncer

Instituto de Atención Pediátrica

2 Universidad de Ciencias Médicas

Abreviaturas: $A D N$, ácido desoxirribonucleico; EMEA, Agencia Europea de Medicamentos; FDA, Administración de Drogas y Alimentos de los Estados Unidos; GMT, títulos medios geométricos; VLP, partículas semejantes al virus; $\mathrm{VPH}$, virus del papiloma humano.

Correspondencia:

Adriano Arguedas Mohs. Apartado postal 607-II50 La Uruca, San José. Email : aarguedas@iped.net cervical.

Descriptores: Virus papiloma humano, VPH, vacuna recombinante, Gardasil ${ }^{\circledR}$, Cervarix ${ }^{\circledR}$

\section{Abstract}

Genital human papillomavirus (HPV) is one of the most common sexually transmitted infections worldwide. Millions of persons are now infected and the lifetime risk of HPV infection exceeds $50 \%$. HPV is approximately $100 \%$ related to the total cases of cervical cancer, $100 \%$ of cervical intraepithelial neoplasias grade 1, 2, and 3; $40 \%$ of vulva, vagina and penile cancer, $100 \%$ of genital warts, $100 \%$ of recurrent respiratory papillomatosis and $12 \%$ of head and neck cancer. Licensed vaccines, Gardasil ${ }^{\circledR}$ and Cervarix ${ }^{\circledR}$, are composed of a noninfectious mixture of HPV type-specific virus-like particles prepared from the L1 proteins produced by recombinant DNA 
ISSN 0001-6002/2008/50/4/203-210 Acta Médica Costarricense, (C2008 Colegio de Médicos y Cirujanos technology and adsorbed in a aluminum-containing adjuvant. Data from several studies of both vaccines reported a range of efficacy of $98,9 \%$ to $100.0 \%$ for the prevention of cervical, vulvar and vaginal intraepithelial neoplasias degrees 2 and 3 , and therefore, cervical, vulvar and vaginal cancer related to the VPH-16/18; in addition to in situ adenocarcinomas and genital warts caused by VPH-16/18/6/11. The effectiveness for both vaccines is maintained for at least 5 years. At the moment no therapeutic effect has been attributed and the vaccine use is prophylactic, nevertheless, vaccination should not be considered as a substitute for cervical cancer screening.

key words: genital human papillomavirus, HPV, recombinant vaccine, Gardasil ${ }^{\circledR}$, Cervarix ${ }^{\circledR}$.

Recibido: 4 de abril de 2008

Aceptado: 6 de mayo de 2008
La infección genital causada por el virus del papiloma humano (VPH) es una enfermedad de transmisión sexual común que afecta a millones de personas. ${ }^{1}$ Se estima que al menos el 50 por ciento de la población sexualmente activa contraerá dicha infección en algún momento de sus vidas. ${ }^{2}$ El virus está asociado aproximadamente a un $100 \%$ de los casos de cáncer cervical, ${ }^{3} 100 \%$ de las neoplasias cervicales intraepiteliales grados $1,2,3 ; ;^{4}$ a un $40 \%$ de los casos de cáncer de vulva, vagina y pene. ${ }^{5}$ Tanto en hombres como en mujeres, el VPH se puede relacionar con el $100 \%$ de las verrugas genitales, ${ }^{6} \mathrm{el} 100 \%$ de las papilomatosis respiratorias recurrentes, ${ }^{7}$ el $90 \%$ en el cáncer anal ${ }^{5}$ y el $12 \%$ en cáncer de cabeza y cuello. ${ }^{5}$

El VPH pertenece a la familia Papillomaviridae, que muestra gran tropismo por las células epiteliales de la piel y membranas mucosas. Contiene un genoma circular de ácido desoxirribonucleico (ADN) de doble cadena localizado dentro de una cápside sin cubierta. ${ }^{8} \mathrm{El}$ genoma viral contiene genes tempranos (E), encargados de la transcripción y replicación viral, así como la interacción con el genoma del huésped, y genes tardíos (L) que codifican las proteínas de la cápside viral. ${ }^{9}$ Las proteínas E6 y E7 del VPH son las principales oncoproteínas responsables de transformar las células epiteliales humanas por medio de la interacción con las proteínas reguladoras del ciclo celular denominadas p53 y pRb. ${ }^{10,11}$

Hasta el momento se han identificado más de 40 genotipos de VPH que afectan el área genital, los cuales son clasificados como de alto y bajo riesgo, de acuerdo con su asociación epidemiológica al cáncer cervical. Los tipos 16, $18,31,33,35,39,45,51,52,56,58,68,69,73$ y 82 son de alto riesgo y han sido detectados en el $99 \%$ de los casos de cáncer cervical, por lo que se consideran necesarios pero no suficientes para la aparición del cáncer y su precursor, la neoplasia cervical intraepitelial. ${ }^{12}$ La mayoría de los adenocarcinomas y carcinomas del cérvix son causados por los tipos 16 y 18; el tipo 16 se asocia principalmente al cáncer de células escamosas de la vulva, vagina, pene y ano. En conjunto todos los anteriores representan el $70 \%$ del total de casos. ${ }^{13,14}$ En el caso de los genotipos de bajo riesgo, destacan los tipos 6 y 11, los cuales están relacionados el $90 \%$ de los diagnósticos de verrugas anogenitales ${ }^{6} \mathrm{y}$ en niños son los responsables de casi la totalidad de casos de papilomatosis respiratoria recurrente. ${ }^{15,16}$ Estudios realizados en niños estimaron una prevalencia del VPH del $1.9 \%$ en la cavidad oral y en la orofaringe, siendo los grupos etários de 1 año o menos y de 16 años o más, los más afectados. ${ }^{17}$ Esta patología puede producir severas obstrucciones de las vías aéreas y dificultades en el habla, por lo que su tratamiento generalmente consiste en frecuentes intervenciones quirúrgicas. ${ }^{18,19}$

Se han documentado respuestas inmunes humorales y celulares contra el virus, pero su correlación con la obtención de inmunidad todavía no es clara. ${ }^{18}$ No todas las personas infectadas desarrollan anticuerpos; en un estudio previamente publicado se determinó que solo entre al 54\% y al $69 \%$ de mujeres con infecciones por VPH 16, 6 ó 18 se les detectaban anticuerpos séricos. ${ }^{20}$ Los anticuerpos mejor caracterizados y más específicos son aquellos dirigidos contra los epitopos conformacionales de la proteína L1 cuando se auto ensambla en una cápside vacía que simula a un virus. ${ }^{18}$

Debido al importante número de individuos en riesgo y a la falta de agentes antivirales efectivos contra el VPH, el desarrollo de vacunas profilácticas se ha convertido en el pilar de las nuevas estrategias para prevenir las infecciones por el VPH particularmente el cáncer cervical. Actualmente están disponibles dos vacunas. Gardasil ${ }^{\mathbb{}}$ es una vacuna recombinante quadrivalente que incluye los tipos 16, 18, 11 y 6 y es producida por los laboratorios Merck; fue aprobada por la Administración de Alimentos y Drogas de los Estados Unidos (FDA por sus siglas en inglés) en junio de 2006 y en Costa Rica se introdujo en el año 2007. Cervarix ${ }^{\circledR}$ es la otra vacuna contra el VPH, producida por los Laboratorios Glaxo SmithKline y ofrece protección contra los tipos 16 y 18. Esta vacuna fue aprobada por la Agencia Europea de Medicamentos (EMEA) en septiembre de 2007; se espera que próximamente sea aprobada por la FDA y el Ministerio de Salud de Costa Rica.

El presente artículo hace una revisión general sobre la epidemiología del VPH, la descripción de las vacunas desarrolladas, recomendaciones para su uso y el impacto que estas podrían ocasionar en el ámbito de la salud pública. 


\section{Epidemiología del VPH}

Al ser el VPH transmitido principalmente por contacto sexual, la gran mayoría de los estudios de prevalencia e incidencia reportan como los principales predictores de la infección, las mediciones de la actividad sexual, y el número de compañeros sexuales. ${ }^{21}$ También existen medios de transmisión del virus menos usuales a la contaminación sexual como por ejemplo, la transmisión que se puede presentar cuando un recién nacido pasa por el canal de parto de una madre contaminada. ${ }^{18}$

La adquisición de la infección ocurre poco tiempo después de iniciar la vida sexual. En aproximadamente el $70 \%$ de las personas que se contaminan con el VPH, la infección desaparece en un período de doce meses, y en el $90 \%$ al transcurrir veinticuatro meses. ${ }^{22}$ Estudios indican que si se adquiere una infección y ésta ha persistido durante 12 a 18 meses se puede esperar que la misma continúe por al menos 6 meses más, inclusive en las mujeres jóvenes quienes tienen la mayor capacidad de eliminar el virus. ${ }^{23}$

Una infección persistente con alguno de los tipos de alto riesgo, principalmente el 16, es el factor de riesgo más importante para desarrollar una displasia o un cáncer cervical. ${ }^{24}$ Otros factores asociados con una mayor incidencia de cáncer cervical incluyen el comportamiento sexual, fumado, multiparidad, edad avanzada, enfermedades de transmisión sexual concomitantes, inmunosupresión y uso prolongado de anticonceptivos orales. ${ }^{25,26}$

Datos reportados por el Centro de Información sobre el Virus del Papiloma Humano y Cáncer Cervical de la Organización Mundial de la Salud indican que en el continente americano existe una población de 336.5 millones de mujeres en riesgo de desarrollar cáncer cervical, y se estima que el $15.6 \%$ de ellas contraerán una infección por el VPH. ${ }^{27}$ En América Central la prevalencia de la infección cervical por VPH en mujeres con citología normal es del $20.5 \%$, mientras que en Norteamérica y Suramérica es del $13.8 \%$ y $14.3 \%$, respectivamente. Cada año en América se diagnostican 86,532 nuevos casos de cáncer de cérvix y 38,436 mujeres mueren a causa de este. ${ }^{27}$

En el caso de Costa Rica, la OMS reporta una prevalencia del VPH del $22.4 \%$ en mujeres con citología normal, el $72.9 \%$ en mujeres con lesiones de grado leve, el $89.8 \%$ en mujeres con lesiones de grado alto y el $97.1 \%$ en aquellas que presentan cáncer cervical. ${ }^{27}$ Según datos emitidos por la Unidad de Estadística del Ministerio de Salud y el Registro Nacional de Tumores de Costa Rica, durante los años 19902000, la incidencia promedio de cáncer de cérvix invasivo presentó una tasa bruta de 17/100 000 mujeres afectadas. ${ }^{28}$ Los cantones de Pococí, Talamanca, Golfito, Los Chiles, Guácimo y Liberia destacan como los más afectados, presentando para los años 1990-1999 tasas ajustadas acumuladas por cada 100,000 mujeres de 45, 41, 39, 39, 38, 38, respectivamente. Para el 2002 la mortalidad por cáncer del cuello uterino disminuyó de manera importante pasando del tercer al cuarto lugar, tanto en incidencia como en mortalidad secundaria a neoplasias malignas en Costa Rica. $^{28}$

Durante la década de los noventa dieron inicio una serie de estudios en 10,049 mujeres en la provincia de Guanacaste, cuyos objetivos eran estimar los factores de riesgo para la infección y las potenciales asociaciones que pudiesen existir entre los tipos específicos del VPH con la neoplasia intraepitelial cervical y el cáncer. ${ }^{29}$ Los resultados de estos estudios epidemiológicos indicaron una prevalencia del VPH del $26.5 \%$ en las mujeres estudiadas. El VPH-16 fue el tipo presente en la mayoría de las lesiones severas, seguido por el $18,58,31,33,45$ y 39, respectivamente. Los tipos no oncogénicos se encontraron en su mayoría en mujeres con citologías normales y fueron el 53, 61, 62, 70 y 71. Este estudio determinó que el comportamiento sexual y el número de compañeros sexuales eran los principales determinantes en la aparición de la infección. Además se menciona la forma de "U" que toma la curva de prevalencia VPH contra la edad de las mujeres estudiadas, en quienes se observa un pico inicial en las menores de 25 años, una disminución en aquellas que se encuentran en edades medias y otro pico en mujeres mayores a los 55 años, destacando la importancia de continuar el tamizaje en mujeres de edad avanzada. ${ }^{30}$

La mayoría de los estudios y publicaciones se enfocan hacia el sexo femenino por la indiscutible asociación entre el VPH y el cáncer cervical, sin embargo, la infección por el virus también es común en los hombres. La prevalencia de VPH en sujetos masculinos varía considerablemente dependiendo de la población en estudio, los sitios anatómicos y el método de recolección de las muestras. Se han reportado cifras tan altas como aquellas en estudios femeninos, pero usualmente se estima una prevalencia menor o igual al $20 \%{ }^{31,32}$ Como dato relevante se ha observado que los hombres circuncidados presentan un menor riesgo de contraer la infección por VPH que los no circuncidados. ${ }^{33}$

\section{Descripción de las vacunas disponibles}

Gardasil $^{\circledR}$ se presenta como una suspensión inyectable en viales de dosis única de $0.5 \mathrm{~mL}$, compuesta por proteínas L1 en forma de partículas no infecciosas similares al virus (VLPs), producidas por tecnología de ADN recombinante mediante el uso de un sistema de expresión que utiliza células derivadas de Sacharomyces cerevisiae. Cada dosis de $0.5 \mathrm{~mL}$ contiene $20 \mu \mathrm{g}$ de proteína L1 de VPH-6, 40 $\mu \mathrm{g}$ de proteína L1 de VPH-11, 40 $\mu$ g de proteína L1 de VPH-16 y $20 \mu \mathrm{g}$ de proteína L1 de VPH-18 adsorbidas en un adyuvante compuesto de sulfato hidroxifosfato de aluminio amorfo. ${ }^{34}$

Cervarix $^{\circledR}$ es una suspensión inyectable producto de tecnología recombinante que utilizan un sistema de expresión con células derivadas de Trichoplusia ni. Cada dosis de $0.5 \mathrm{~mL}$ está compuesta por $20 \mu \mathrm{g}$ de proteína L1 de VPH-16 y $20 \mu \mathrm{g}$ de proteína L1 de VPH-18 en forma de 
VLPs adsorbidas en el adyuvante llamado ASO4, el cual está compuesto por hidróxido de aluminio y monofosforil lípido A. ${ }^{35}$ (Cuadro 1)

Los viales de ambas vacunas deben mantenerse en cadena de frío $\left(2^{\circ} \mathrm{C}-8^{\circ} \mathrm{C}\right)$ durante todo su proceso de distribución y almacenamiento; además, siempre debe conservarse dentro de su empaque original para proteger la vacuna de la luz directa con el fin de garantizar su eficacia y estabilidad. ${ }^{34,35}$

El mecanismo de acción de ambas vacunas se basa en la capacidad de las VLPs de inducir altos niveles de anticuerpos neutralizantes IgG en medio vaginal. ${ }^{36,37}$ Puesto que las VLPs, no contienen ADN viral, no pueden infectar células, reproducirse o causar enfermedad. Los estudios en animales han mostrado que la eficacia de las vacunas de VLP L1 está mediada fundamentalmente por el desarrollo de una respuesta inmune humoral. ${ }^{38}$

\section{Eficacia, inmunogenicidad y seguridad de las vacunas disponibles}

La eficacia de Gardasil ${ }^{\circledR}$ fue evaluada en 4 estudios fases II y III randomizados, doble ciego y controlados con placebo, que juntos analizaron una población superior a 20,500 mujeres con edades entre los 16 y 26 años, provenientes de América, Asia, Europa y Oceanía. ${ }^{39-42}$ La eficacia de los estudios en la población según el protocolo (sujetos que recibieron al menos una dosis y fueron PCR negativos y seronegativos a los tipos VPH 6,11,16 y/o 18 en el día 1) fue de un 98.8\% (95\% IC: 93.3-100.0) para la prevención de neoplasias cervicales intraepiteliales grado 2 , grado $3 \mathrm{o}$ adenocarcinoma in situ, un 100\% (95\% IC: 41.7-100.0) para las neoplasias vulvares intraepiteliales grados 2 ó 3 y un 100\% (95\% IC: $30.9-100.0)$ para las neoplasias vaginales intraepiteliales grados 2 ó 3 relacionados con el VPH-16/18; además de una eficacia del 98.8\% (95\% IC: 95.4-99.9) para las verrugas genitales causadas por VPH-16/18/ 6/11. ${ }^{9}$ Mientras que la eficacia en la población general (sujetos que recibieron al menos una dosis sin tomar en cuenta su estado de VPH basal en el día 1) fue de un 39.0\% (95\% IC: 23.3 51.7) para la prevención de neoplasias cervicales intraepiteliales grado 2, grado 3 o adenocarcinoma in situ, un 69.1 (95\% IC: 29.8-87.9) para las neoplasias vulvares y vaginales intraepiteliales grados 2 o 3 y una eficacia del $68.5 \%$ (95\% IC: $57.5-77.0)$ para las verrugas genitales causadas por VPH-16/18/6/11. ${ }^{9}$ (Cuadro 2) La mayoría de las neoplasias y verrugas reportadas en este grupo se encontraban presentes desde el día 1. La inmunogenicidad se evaluó en mujeres de 9 a 26 años y en hombres de 9 a 15 años de edad. Cerca del $99.8 \%$ de los sujetos vacunados resultaron anti-VPH 16/18/6/11 seropositivos un mes luego de la tercera dosis, desarrollando niveles de anticuerpos séricos significativamente superiores comparados con el grupo placebo. ${ }^{43}$ En general, los títulos serológicos antiVPH alcanzaron su máximo al séptimo mes luego de la primera dosis y después disminuyeron alcanzando concentraciones estables hasta por 5 años ${ }^{44}$ Los resultados obtenidos en el grupo de adolescentes y preadolescentes de 9 a 15 años de edad mostraron títulos medios geométricos (GMT) al mes 18, dos veces superiores comparados a los GMT obtenidos en el grupo de mujeres adultas durante los estudios fase III donde se determinó la eficacia; debido a que esta no se puede evaluar en sujetos vírgenes, la información anterior hace posible inferir que la vacuna es eficaz en niñas de esta edad. ${ }^{43}$

Se cuenta con estudios que demuestran que Gardasi ${ }^{\circledR}$ es inmunogénico y bien tolerado en sujetos masculinos. Respuestas anti-VPH al mes 7 en niños de 9 a 15 años de edad fueron superiores a aquellas encontradas en las niñas de la misma edad y no fueron inferiores a las presentadas por las mujeres de 16 a 26 años. ${ }^{43}$ Sin embargo, no se ha determinado si la vacuna pueda proveer protección a la población masculina de igual forma que a la femenina, ya que la respuesta inmunológica local genital va a depender de diversos factores como lo son la anatomía y fisiología del sujeto. Varias vacunas poseen eficacias comparables en hombres y mujeres, no obstante se puede mencionar el caso de la vacuna contra el virus del herpes simple tipo 2, otra infección viral de transmisión sexual, que resultó efectiva en mujeres pero no así en hombres, lo cual plantea la duda de si se podría presentar el mismo problema con la vacuna contra el VPH. ${ }^{45}$

\begin{tabular}{|lcc|}
\hline \multicolumn{2}{|c|}{ Cuadro 1. Características generales de las vacunas contra el VPH ${ }^{34,46}$} \\
\hline & Gardasil $^{\circledR}$ & Cervarix $^{\circledR}$ \\
Tipos de VPH presentes & $16,18,11,6$ & 16,18 \\
& $40 \mu \mathrm{g}$ de VPH-16 & $20 \mu \mathrm{g}$ de VPH-16 \\
Cantidad de proteína L1 en cada dosis de 0.5mL & $20 \mu \mathrm{g}$ de VPH-18 & $20 \mu \mathrm{g}$ de VPH-18 \\
& $40 \mu \mathrm{g}$ de VPH-11 & \\
Población dirigida & $20 \mu \mathrm{g}$ de VPH-6 & Mujeres de 10 a 25 años \\
& Mujeres de 9 a 26 años & Mujeres de 10 a 45 años \\
Esquema de vacunación en meses & Hombres de 9 a 15 años & $0-1-6$ \\
\hline
\end{tabular}


Cuadro 2 . Eficacia de las vacunas contra el VPH para la prevención de neoplasias intraepiteliales y verrugas genitales ${ }^{9,46}$

\begin{tabular}{|c|c|c|}
\hline & 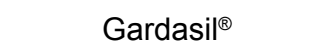 & Cervarix $^{\circledR}$ \\
\hline $\begin{array}{l}\text { Neoplasias cervicales intraepiteliales } \\
\text { grado } 2 \text { o } 3\end{array}$ & $\begin{array}{c}98.8 \%^{\mathrm{a}} \\
(95 \% \text { IC: } 93.3-100.0) \\
39.0 \% \mathrm{~b} \\
(95 \% \text { IC: } 23.3-51.7)\end{array}$ & $\begin{array}{c}100 \% \\
(97.9 \% \text { IC: } 74.2-100.0)\end{array}$ \\
\hline $\begin{array}{l}\text { Neoplasias vulvares intraepiteliales } \\
\text { grado } 2 \circ 3\end{array}$ & $\begin{array}{c}100 \%^{\mathrm{a}} \\
(95 \% \text { IC: } 41.7-100.0) \\
69.1 \%{ }^{\mathrm{b}} \\
(95 \% \text { IC: } 29.8-87.9)\end{array}$ & $\begin{array}{c}\text { No hay } \\
\text { datos disponibles }\end{array}$ \\
\hline $\begin{array}{l}\text { Neoplasias vaginales intraepiteliales } \\
\text { grado } 203\end{array}$ & $\begin{array}{c}100 \%^{\mathrm{a}} \\
(95 \% \text { IC: } 30.9-100.0) \\
69.1 \% \%^{\mathrm{b}} \\
(95 \% \text { IC: } 29.8-87.9)\end{array}$ & $\begin{array}{c}\text { No hay } \\
\text { datos disponibles }\end{array}$ \\
\hline Verrugas genitales & $\begin{array}{c}98.8 \%^{\mathrm{a}} \\
(95 \% \text { IC: } 95.4-99.9) \\
68.5 \% \mathrm{~b} \\
(95 \% \text { IC: } 57.5-77.0)\end{array}$ & $\begin{array}{c}\text { No hay } \\
\text { datos disponibles }\end{array}$ \\
\hline
\end{tabular}

a Población según protocolo, ${ }^{\text {b }}$ Población general.

Muchos países han decido aprobar la aplicación de la vacuna en hombres a pesar de que no se cuentan aún con datos sobre la eficacia de la misma en dicha población. Pero se justifican con otros hechos tales como que los hombres también pueden desarrollar enfermedades relacionadas con los VPH-16/18/ 6/11 y además son los principales vectores para la transmisión del virus a las mujeres.

La eficacia de Cervarix ${ }^{\circledR}$ fue evaluada en 19,778 mujeres con edades entre los 15 y 25 años, en dos estudios fase II y uno fase III randomizados, doble ciego y controlados con placebo. Se determinó una eficacia del 100\% (97.9\% IC: 74.2-100.0) para la prevención de neoplasias cervicales intraepiteliales grado 2 y 3 asociadas a VPH-16/18. ${ }^{46} \mathrm{La}$ inmunogenicidad inducida por esta vacuna se determinó en 5,303 mujeres con edades entre los 10 a los 55 años. Durante los estudios clínicos el $99.9 \%$ de los sujetos inicialmente seronegativos mostraron seroconversión para los tipos HPV16/18 un mes después de la tercera dosis, además, para ese mismo período se pudo detectar en las secreciones cervico vaginales, anticuerpos HPV-16 y 18, en aproximadamente el $80 \%$ y el $68 \%$ de las mujeres respectivamente; aún no se ha definido el papel que la actividad y los títulos de los anticuerpos locales puedan tener. ${ }^{46}$ En los estudios de seguimiento se encontró que los GMT para ambos tipos de HPV presentan un máximo al mes 7, para luego disminuir y alcanzar niveles estables hasta el mes 64, período del cual se tienen datos hasta el momento. Al mes 64 (quinto año) más del $98 \%$ de las mujeres permanecieron seropositivas para ambos antígenos. ${ }^{46}$ Un dato importante de destacar es que los datos publicados indican que las vacunas basadas en VLPs de L1 no presentan ningún efecto terapéutico, son de tipo profilácticas y brindan protección contra los episodios directamente relacionados con los tipos de VPH que contienen. ${ }^{47}$ Sin embargo estudios indican que la vacuna produce algún tipo de protección cruzada contra los tipos VPH 31 y $45 .{ }^{38}$

La seguridad de Gardasil ${ }^{\circledR}$ se evaluó en 7 estudios diferentes. Se vacunaron alrededor de 11,778 personas entre los 9 y 26 años, y 9600 participantes recibieron placebo. La vacunación en general fue bien tolerada: menos del $0,1 \%$ de los pacientes reportaron inconvenientes para continuar con la vacunación. Las proporciones de sujetos que presentaron efectos adversos serios fueron comparables entre los grupos vacunados y los del placebo. Los efectos locales mayormente reportados fueron dolor en el sitio de aplicación (83.9\%), hinchazón (25.4\%) y eritema (24.7\%), los cuales fueron clasificados de intensidad leve a moderada. Fiebre igual o superior a los $38^{\circ} \mathrm{C}(13.0 \%)$, náuseas $(6.7 \%)$, nasofaringitis $(6.4 \%)$, mareo $(4.0 \%)$ y diarrea $(3.6 \%)$ fueron los efectos sistémicos con más reportes en el grupo vacunado. Durante los estudios se reportaron 18 muertes, de las cuales ninguna se relacionó con la administración de la vacuna. ${ }^{4,12,25}$

Para determinar la seguridad de Cervarix ${ }^{\circledR}$ se analizó información proveniente de 11 estudios que en total incluyeron a 16142 mujeres vacunadas y 13811 sujetos recibieron placebo. Los efectos adversos reportados incluyen dolor en el sitio de aplicación (78.0\%), eritema (29.6\%), hinchazón (25.8\%), fatiga (33.1\%), síntomas gastrointestinales (12.9\%), cefalea $(29.5 \%)$ y mialgia $(28.1 \%) .{ }^{46}$

Tanto Cervarix ${ }^{\circledR}$ como Gardasil ${ }^{\circledR}$ fueron clasificadas como categoría B en el embarazo, ya que estudios realizados en ratas no mostraron evidencia de que pudiera afectar la fertilidad o dañar al feto. Sin embargo, esto no se puede afirmar con certeza en el caso de los humanos, por lo tanto, 
no se recomienda administrar la vacuna en mujeres embarazadas, debido a la falta de información en este tipo de población. ${ }^{34,46} \mathrm{Si}$ se ha vacunado a una mujer embarazada no es necesario realizar intervención alguna, pero se deben posponer las dosis restantes hasta haber completado el período de gestación. ${ }^{18}$ No es claro si los antígenos de la vacuna o los anticuerpos inducidos por esta se excretan en la leche materna. Debido a que un gran número de medicamentos se excretan por esta vía, se deben tomar precauciones cuando la vacuna es administrada a una mujer en período de lactancia..$^{34,46}$

\section{Dosis y administración}

La administración de la vacuna en forma ideal, pero no necesariamente, se debe realizar antes de que la persona inicie su vida sexual. Aquellas mujeres que ya han sido expuestas e incluso infectadas con alguno de los tipos VPH presentes en la vacuna, pueden ser vacunadas aunque el beneficio podría ser menor. ${ }^{18}$

Para la aplicación de la vacuna no es necesario realizar pruebas citológicas o serológicas, sin embargo, el haber recibido la vacuna no implica que se deba restar importancia a las pruebas de tamizaje para la detección del VPH, ya que eventualmente las mujeres vacunadas podrían contraer la infección por algún tipo carcinógeno o no, que no se encuentra incluido en la vacuna.

El Comité Asesor sobre las Prácticas de Inmunización de los Estados Unidos (ACIP por sus siglas en inglés) recomienda la aplicación de Gardasil ${ }^{\circledR}$ en forma rutinaria para mujeres con edades entre los 11 y 12 años, sin embargo, la vacunación puede iniciarse desde los 9 años de edad. Se recomienda, además, la vacunación de mujeres entre los 13 y 26 años que no han sido inmunizadas previamente, o que no completaron el esquema correspondiente. El uso de esta vacuna en hombres aún no está avalado en los Estados Unidos, ${ }^{18,34,43}$ pero en múltiples países europeos y latinoamericanos, incluido Costa Rica, la vacuna está aprobada para administrarse a sujetos masculinos con edades de 9 a 15 años. $^{48}$

La dosis de la vacuna Gardasil ${ }^{\circledR}$ es de $0,5 \mathrm{~mL}$, aplicada intramuscularmente en el músculo deltoides o en el muslo anterior superior. Se aplica con un esquema de dosificación 0-2-6. La segunda aplicación se realiza 2 meses después de la primera, con un intervalo mínimo 4 semanas entre dosis. La tercera, dosis se administra 4 meses luego de la segunda con un intervalo mínimo de 12 semanas. Si el esquema de vacunación se ve interrumpido, no es necesario iniciarlo de nuevo. En el caso de que fuese suspendido luego de la primera dosis, la segunda deberá ser aplicada lo más pronto posible, para posteriormente administrar la tercera, luego de haber transcurrido un período mínimo de 12 semanas entre ellas. Si solo la tercera dosis es retrasada, se debe administrar lo antes posible. ${ }^{18,34}$
Hay estudios que avalan la administración concomitante de Gardasil ${ }^{\circledR}$ con la vacuna de hepatitis B. ${ }^{18,34}$ Al no ser una vacuna de virus vivos, se supone que esta no interfiere con la eficacia o seguridad de otras vacunas. La vacuna contra VPH se puede administrar junto con la vacuna de tétano difteria y tosferina y la vacuna meningocóccica conjugada quadrivalente, en diferentes jeringas y en distintos sitios anatómicos. $^{18}$

Cervarix $^{\circledR}$ está aprobado para administrarse en mujeres de 10 a 25 años con el esquema 0-1-6 (la segunda aplicación se administra un mes luego de la primera dosis y la tercera 6 meses después de la primera dosis) ${ }^{34}$ En Australia entidades sanitarias de dicho país autorizaron su uso para mujeres con edades de 10 a 45 años; sin embargo, esta vacuna no está todavía recomendada para su uso en Costa Rica. ${ }^{49}$ Hasta el momento no se cuenta con datos suficientes para que las autoridades competentes autoricen el uso de tal vacuna en la población masculina. ${ }^{46}$

Las vacunas, al ser de tipo no infecciosas, pueden administrarse a personas con inmunodeficiencia causada por enfermedad o por medicamentos, pero tanto la respuesta inmune como la eficacia de la vacunas podrían ser menores a las obtenidas en una persona inmunocompetente. ${ }^{18}$ En caso de afecciones agudas menores como diarrea o infecciones leves de las vías respiratorias superiores con o sin fiebre, los pacientes pueden ser vacunados.

\section{Impacto esperado de la vacunación}

Hasta la fecha se han desarrollado diversos modelos para evaluar el impacto clínico y económico de la vacunación contra el VPH . Los resultados varían dependiendo del modelo aplicado, sin embargo, la mayoría concuerda que de ser posible una aplicación generalizada de la vacuna, se podría alcanzar una marcada disminución de la incidencia de cáncer cervical. Utilizando el modelo de Markov, se ha estimado que si se vacunara una cohorte de 1988600 mujeres de 12 años de edad, se podría reducir el riesgo de presentar cáncer cervical entre un $20 \%$ y un $66 \% .^{50,51}$ Sin embargo, estas aproximaciones dependen de la eficacia y duración de la protección brindada por la vacuna, datos que por el momento son limitados. ${ }^{52}$ Otros modelos proyectan disminuciones en anormalidades y precursores de cáncer de cérvix detectados en los exámenes de Papanicolau, debido a la vacunación hasta de un $21 \%{ }^{44}$ En el caso de las verrugas genitales, se ha publicado un único estudio que estima un descenso de hasta un $97 \%$ en la incidencia de esta enfermedad si la vacuna se aplicara en forma extensiva tanto en sujetos femeninos como masculinos. ${ }^{53}$ No se cuentan por el momento con publicaciones que evalúen el impacto de la vacunación sobre las otras enfermedades asociadas al VPH como el cáncer de vulva, vagina, ano y pene, la papilomatosis respiratoria recurrente y algunas neoplasias orofaríngeas. ${ }^{54}$

Diversos análisis de costos-efectividad han buscado cuantificar las implicaciones financieras de la vacunación en 
diversas regiones alrededor del mundo. Gran parte de estos estudios fueron realizados en los Estados Unidos de Norteamérica, los cuales estiman un rango de $\$ 3,000$ a $\$ 24,000$ de inversión en dicho país por cada año de vida ajustado según la calidad ganado, un valor que se encuentra dentro del rango de otras intervenciones preventivas que están actualmente en práctica. ${ }^{53}$

\section{Conclusión}

A pesar de ser un biológico de reciente aprobación, las vacunas recombinantes contra el VPH han demostrado ser seguras y eficaces para prevenir las infecciones y neoplasias anogenitales asociadas con el virus. Se espera que con la inclusión en las vacunas de los tipos más frecuentes causantes de las manifestaciones de mayor impacto en el nivel sanitario, junto con los diferentes programas y campañas de prevención y las pruebas de tamizaje para el cáncer de cérvix, se presente una importante disminución en la incidencia de las enfermedades relacionadas con el VPH y, en especial, con el cáncer del cuello uterino, una de las principales causas de mortalidad femenina. Aún existen varias interrogantes de aspecto biológico y abundantes retos en el área de la implementación que se deben de enfrentar, por lo que se debe hacer un mayor énfasis de mantener la vigilancia sobre aspectos de seguridad y eficacia, así como realizar estudios de costo y beneficio que respalden la creciente introducción de la vacuna en los sistemas de salud mundial.

\section{Referencias}

1. World Health Organization. Human papillomavirus infection and cervical cancer. 2004.

2. En: http://www.who.int/vaccine _research/diseases/hpv/en/. Consultado el 1 de febrero de 2008.

3. Centros para el Control y la Prevención de Enfermedades (CDC). Genital HPV Infection-CDC Fact Sheet. 2004.

En: http://www.cdc.gov/std/ HPV/ STDFact-HPV.htm.

Consultado: el 1 de febrero de 2008.

4. Clifford G, Smith J, Aguado T, Franceschi S. Comparison of HPV type distribution in high-grade cervical lesions and cervical cancer: a meta-analysis. Br J Cancer 2003; 89:101-5.

5. Clifford G, Rana R, Franceschi S, Smith J, Gough G, Pimenta J. Human papillomavirus genotype distribution in low-grade cervical lesions: comparison by geographic region and with cervical cancer. Cancer Epidemiol Biomarkers Prev 2005; 14:1157-64.

6. Parkin D. The global health burden of infection-associated cancers in the year 2002. Int J Cancer 2006; 118:3030-44.

7. Greer C, Wheeler C, Ladner M, Beutner K, Coyne MY, Liang H, et al. Human papillomavirus (HPV) type distribution and serological response to HPV type 6 virus-like particles in patients with genital warts. J Clin Microbiol 1995; 33:2058-63.
8. Wiatrak B, Wiatrak D, Broker T, Lews L. Recurrent respiratory papillomatosis: a longitudinal study comparing severity associated with human papilloma viral types 6 and 11 and other risk factors in large pediatric population. Laryngoscope 2004; 114:1-23.

9. Jansen K, Shaw A. Human papillomavirus vaccines and prevention of cervical cancer. Annu Rev Med 2004; 55:319-31.

10. Barr E, Tamms G. Quadrivalent Human Papillomavirus Vaccine. Clin Infect Dis 2007; 45:609-17.

11. Ball E. Virus Papiloma Humano. Biología Molecular, Genética y Mecanismo Oncogénico, Parte II. Derm Venez 1999; 37:5-10.

12. Adams M, Borysiewicz L, Fiander A, Man S, Jasani B, Navabi H, et al. Clinical studies of human papilloma vaccines in pre-invasive and invasive cancer. Vaccine 2001; 19:2549-2556.

13. Walboomers J, Jacobs M, Manos M, Bosch FX, Kummer JA, Shah $\mathrm{KV}$ et al. Human papillomavirus is a necessary cause of invasive cervical cancer worldwide. J Pathol 1999; 189:12-9.

14. Bosch F, de Sanjosé S. Human papillomavirus and cervical cancerburden and assessment of casuality. J Natl Cancer Inst Monogr 2003; 31:3-13.

15. Muñoz N, Bosch F, de Sanjosé S, Herrero R, Castellsagué X, Shah KV et al. Epidemiologic classification of human papillomavirus types associated with cervical cancer. N Engl J Med 2003; 348:518-27.

16. Draganov $\mathrm{P}$, Todorov S, Todorov I, Karcher T, Kalvatchev Z. Identification of HPV DNA in patients with juvenile-onset recurrent respiratory papillomatosis using SYBR Green real-time PCR. Int J Pediatr Otorhinolaryngol 2006; 70:469-73.

17. Major T, Szarka K, Sziklai I, Gergely L, Czeglédy J. The characteristics of human papillomavirus DNA in head and neck cancers and papillomas. J Clin Pathol 2005;58:51-5.

18. Smith E, Swarbavel S, Ritchie J, Wang D, Haugen T, Turek L. Prevalence of human papillomavirus in the oral cavity/oropharynx in a Large population of children and adolescents. Pediatr Infect Dis J 2007; 26:836-40.

19. Markowitz L, Dunne E, Saraiya M, Lawson H, Chesson H, Unger E. Quadrivalent Human Papillomavirus Vaccine: Recommendations of the advisory committee on inmunization practices (ACIP). MMWR: Morbidity \& Mortality Weekly Report 2007; 56 (RR-2):1-24.

20. Alexander K. Diagnosis and management of human papillomavirus infections. Pediatr Infect Dis J 2005; 24:1007-8.

21. Carter J, Koutsky L, Hughes J, Lee SK, Kuypers J, Kiviat N, Galloway DA et al. Comparison of human papillomavirus types 16,18 , and 6 capsid antibody responses following incident infection. J Infect Dis 2000; 181:1911-9.

22. Ho G, Bierman R, Beardsley L, Chang C, Burk R. Natural history of cervicovaginal papillomavirus infection in young women. N Engl J Med 1998; 338:423-8.

23. Franco E, Villa L, Sobrinho J, Prado JM, Rousseau MC, Désy M, Rohan TE. Epidemiology of adquisition and clearance of cervical human papillomavirus infection in women from a high-risk area for cervical cancer. J Infect Dis 1999; 180:1415-23.

24. Plummer M, Schiffman M, Castle P, Maucort-Boulch D, Wheeler C. A 2 Year prospective study of human papillomavirus persistence among women with a cytological diagnosis of atypical squamous cells of undetermined significance or low-grade squamous intraepithelial lesion. J Infect Dis 2007; 195:1582-9.

25. Wheeler C, Hunt W, Schiffman M, Castle P. Human papillomavirus genotypes and the cumulative 2 -year risk of cervical precancer. $\mathrm{J}$ Infect Dis 2006; 194:1291-9.

26. Zelmanowicz A, Schiffman M, Herrero R, Goldstein AM, Sherman ME, Burk RD et al. Family history as a co-factor for adenocarcinoma and squamous cell carcinoma of the uterine cervix: results from two studies conducted in Costa Rica and the United States. Int J Cancer 2005; 116:599-605. 
27. Bosch F, Lorincz A, Muñoz N, Meijer C, Shah K. The causal relation between human papillomavirus and cervical cancer. J Clin Pathol 2002; 55: 244-265.

28. Organización Mundial de la Salud y Centro de Información de VPH y Cáncer Cervical (WHO/ICO). Summary report on HPV and cervical cancer statistics in Spain. 2007.

En: http://www.who.int/hpvcentre. Consultado el 1 de febrero de 2008.

29. Ministerio de Salud de Costa Rica. Incidencias de cáncer 1999-2003: Cáncer de cérvix. 2005.

En: http://www.ministeriodesalud.go.cr/cancer/cancercervix.pdf. Consultado el 3 de febrero de 2008.

30. Herrero R, Castle P, Schiffman M, Bratti M, Hildesheim A, Morales J, et al. Epidemiologic profile of type-specific human papillomavirus infection and cervical neoplasia in Guanacaste, Costa Rica. J Infect Dis 2005 ; 191:1796-1807.

31. Castle P, Schiffman M, Herrero R, Hildesheim A, Rodríguez A, Bratti $\mathrm{M}$, et al. A prospective study of age trends in cervical human papillomavirus acquisition and persistence in Guanacaste, Costa Rica. J Infect Dis 2005; 191:1808-16.

32. Hernandez B, Wilkens L, Zhu X, McDuffie K, Thompson P, Shvetsov $\mathrm{Y}$, et al. Circumcision and human papillomavirus infection in men: a site-specific comparison. J Infect Dis 2008; 197:787-94.

33. Dunne E, Nielson C, Stone K, Markowitz L, Giuliano A. HPV Infection among men: a systematic review of the literature. J Infect Dis 2006; 194:1044-57.

34. Partridge J, Koutsky L. Genital human papillomavirus infection in men. Lancet Infect Dis 2006; 6:21-31.

35. Gardasil: Quadrivalent Human Papillomavirus (Types 6, 11, 16, 18) Recombinant Vaccine Product Information. Whitehouse Station, NJ. Merck \& Co., Inc.

En: http://www.merck.com/product/usa/picirculars/g/gardasil/ gardasil_pi.pdf. Consultado el 3 de febrero de 2008.

36. Agencia Europea de Medicamentos (EMEA). Informe Público Europeo de Evaluación (EPAR) Cervarix. 2007.

En: www.emea.europa.eu/ humandocs/PDFs/EPAR/cervarix/H-721es1.pdf. Consultado el 3 de febrero de 2008.

37. Stanley M, Lowy D, Frazer I. Prophylactic HPV vaccines: underlying mechanisms. Vaccine 2006; 24:S106-13.

38. Lowy D, Schiller J. Prophylactic human papillomavirus vaccines. J Clin Invest 2006; 116:1167-73.

39. Sharma R, Sharma C. Quadrivalent human papillomavirus recombinant vaccine: The first vaccine for cervical cancers. J Cancer Res Ther 2007; 3:92-95.

40. Garland S, Hernández M, Wheleer C, Pérez G, Harper D, Leodolter S, et al. Quadrivalent vaccine against human papillomavirus to prevent anogenital diseases. N Engl J Med 2007; 356:1928-43.

41. Future II Study Group. Quadrivalent vaccine against human papillomavirus to prevent high-grade cervical lesions. N Engl J Med 2007; 356:1915-27.
42. Future II Study Group. Prophylactic efficacy of a quadrivalent HPV vaccine in women with virologic evidence of HPV infection. J Infect Dis 2007; 196: 1438-46.

43. Ault K; Future II Study Group. Effect of prophylactic human papillomavirus $\mathrm{L} 1$ virus-like particle vaccine on risk of cervical intraepithelial neoplasia grade 2 , grade 3 , and adenocarcinoma in situ: a combined analysis of four randomized clinical trials. Lancet 2007; 369:1861-8.

44. EMEA. European Public Assessment Report. Gardasil Scientific Discussion. 2006.

En: $\quad$ www.emea.europa.eu/humandocs/PDFs/EPAR/gardasil/ 070306en6.pdf. Consultado el 3 de febrero de 2008.

45. Stanberry L, Spruance SL, Cunningham AL, Bernstein DI, Mindel A, Sacks S, Tyring S, et al. Glycoprotein-D-adjuvant vaccine to prevent genital herpes. N Engl J Med 2002; 347:1652-61.

46. Olson S, Villa L, Costa R, et al. Induction of immune memory following administration of a prophylactic quadrivalent human papillomavirus (HPV) types 6/11/16/18 L1 virus-like particle vaccine. Vaccine 2007; 25:4931-39.

47. EMEA. European Public Assessment Report. Cervarix Scientific Discussion. 2007.

Consultado en: www.emea.europa.eu/humandocs/PDFs/EPAR cervarix/H-721-en6.pdf._itado el 12 de febrero de 2008.

48. Hildesheim A, Herrero R, Wacholder S, Rodriguez AC, Solomon D, Bratti MC et al. Effect of human papillomavirus 16/18 L1 viruslike particle vaccine among young women with preexisting infection: a randomized trial. JAMA 2007; 298:743-53.

49. EMEA. European Public Assessment Report. EPARs for authorised medicinal products for human use. 2007.

En: http://www.emea.europa.eu/humandocs/Humans/EPAR/gardasil/ gardasil.htm. Consultado el 02 de abril de 2008.

50. Australian Therapeutic Goods Administration. 2007. Australian Drug Evaluation Committee - 251st meeting recommendations.

En: http://www.tga. gov.au/docs/html/adec/adec0251.htm. Consultado el 18 de marzo de 2008.

51. Sanders G, Taira A. Cost-effectiveness of a potential vaccine for human papillomavirus. Emerg Infect Dis 2003; 9:37-48.

52. Goldie S, Kohli M, Grima D, Weinstein MC, Wright TC, Bosch FX, Franco E. Projected clinical benefits and cost-effectiveness of a human papillomavirus 16/18 vaccine. J Natl Cancer Inst 2004; 96:604-15.

53. Brisson M, Van de Velde N, De Wals P, Boily M. Estimating the number needed to vaccinate to prevent diseases and death related to human papillomavirus infection. CMAJ 2007; 177:464-8.

54. Elbasha E, Dasbach E, insinga R. Model for assessing human papillomavirus vaccination strategies. Emerg Infect Dis 2007; 13:28-41.

55. Dempsey A, Freed G. Human papillomavirus vaccination: expected impacts and unresolved issues. J Pediatr 2008; 152:305-9. 